\title{
Case Series on Contribution of CT and MRI in Diagnosis of Brain Death
}

\author{
Tanushree R Kamath ${ }^{1}$, Ram Shenoy Basti ${ }^{2}$, Mahesh ${ }^{3}$ \\ ${ }^{1}$ Resident, Department of Radiodiagnosis, ${ }^{2}$ Professor, Department of Radiodiagnosis, ${ }^{3}$ Senior Resident, Radiodiagnosis, \\ Father Muller Medical College, Hospital, Mangalore, Karnataka, India
}

Corresponding author: Dr Tanushree R Kamath, 201-Poojan Pearl Apratments, Jodumatta Street, Mangalore - 575001, India

DOI: http://dx.doi.org/10.21276/ijcmsr.2019.4.3.52

How to cite this article: Tanushree R Kamath, Ram Shenoy Basti, Mahesh. Case Series on contribution of CT and $\mathrm{MRI}$ in diagnosis of brain death. International Journal of Contemporary Medicine Surgery and Radiology. 2019;4(3):C234-C236.

\section{A B S T R A C T}

Introduction: Brain death is the irreversible cessation of function of the brain, including the brain stem; the diagnosis of brain death is primarily based on clinical criteria, usually supported be confirmatory tests. There are particular circumstances when clinical tests cannot be applied hence other ancillary tests come to play.CT and MRI pose as noninvasive methods and with the presence of specific findings can aid in diagnosis with MRI having an added advantage of demonstrating the absence of intracranial flow, thus confirming brain death.

Case report: It includes three cases including two MRI and one CT which shows typical radiological findings of brain dead like diffuse sulcal effacement, tonsillar herniation, loss of flow voids and marked diffuse ADC drop.

Conclusion: There is a need to create awareness and to exploit the usefulness of specific radiological findings of brain death in scenarios where objective documentation is needed or to shorten the period of observation required before organ donation. It also helps to reduce the time taken to confirm diagnosis and helps avoid unnecessary ICU stays which are a financial burden. The radiologist role will be to notify the clinician regarding the same which will help in decision making and avoid overburdening the patients family and its consequences to follow in present scenario.

Keywords: $\mathrm{CT}$, MRI, Brain Death

\section{INTRODUCTION}

Brain death is the irreversible cessation of function of the brain, including the brain stem; the diagnosis of brain death is primarily based on clinical criteria, usually supported be confirmatory tests. ${ }^{1}$ Confirmatory tests come to play when clinical testing is not possible. It helps to reduce the time taken to confirm diagnosis, helps to avoid unnecessary ICU stays which are a financial burden. It helps to expedite organ donation and also imaging can be stored as objective documentation. CT and MRI pose as noninvasive methods and with the presence of specific findings can aid in diagnosis with MRI having an added advantage of demonstrating the absence of intracranial flow, thus confirming brain death.

\section{CASE 1}

20 year old patient presented with history of fever with chills since 5 days and vomiting, bleeding from gums, hematemesis. Lab investigations were dengue positivity with thrombocytopenia, coagulopathy, deranged LFT and RFT. Patient had worsening of sensorium and EEG showed diffuse slowing of waves.

CT brain showed diffuse sulcal effacement, tonsillar herniation for 12(figure-1).

\section{CASE 2}

22 year old lady was brought with history of fever, myalgia, status epilepticus. On examination she was seen to have respiratory acidosis, thrombocytopenia, coagulopathy. USG showed a 5 week gestational sac. MRI was advised. It showed Diffuse increased T2/FLAIR signal of cerebral cortex suggestive of edema, tonsillar herniation, loss of flow voids in bilateral internal carotid arteries and marked diffuse ADC drop (fig-2).

\section{CASE 3}

A full term vaginal delivery baby with history of meconium stained amniotic fluid and asphyxia presented with

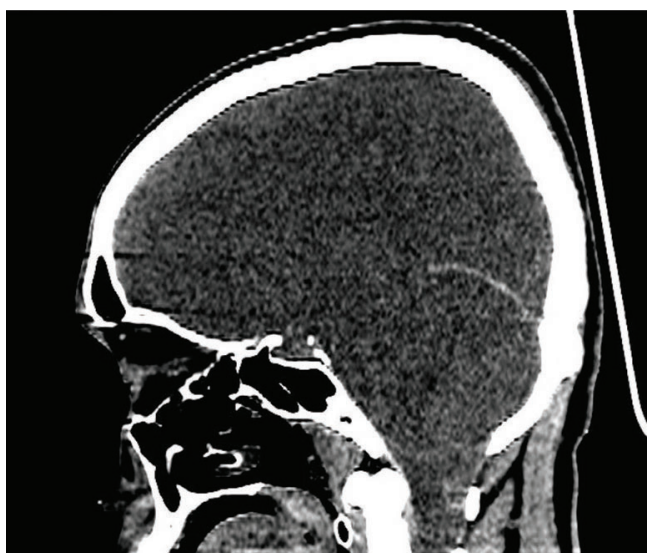

Figure-1: Case 1 CT brain 


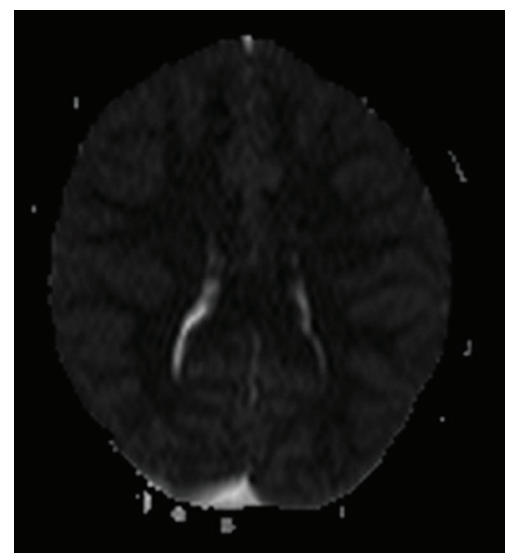

Figure-2: Case 2: MRI shows diffuse drop in ADC

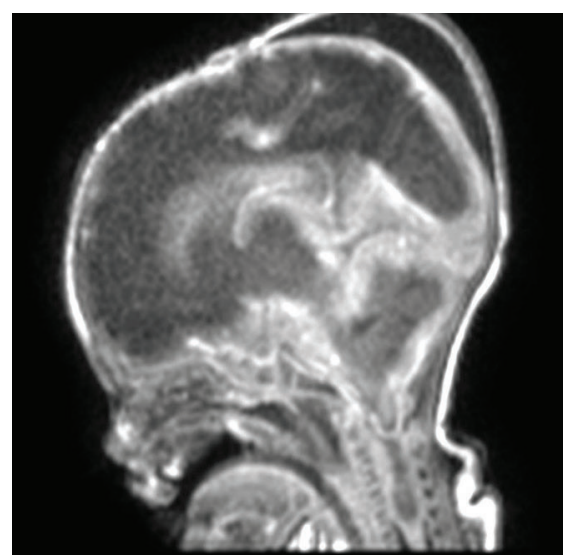

Figure-3: MRI was advised which showed diffuse cerebral edema (case 3)

convulsions, cyanosis and tachypnea. ECHO showed moderate ASD, small PDA, severe TR, dilated RA, RV.MRI was advised which showed diffuse cerebral edema, tonsillar herniation and marked ADC drop (fig-3).

\section{DISCUSSION}

The irreversible cessation of function of brain is due to cerebral circulatory arrest. The reason for cessation of cerebral blood flow in brain injury is cerebral edema as the skull bones limit the expansion resulting in raised intracranial pressure. When raised ICP exceeds the mean arterial pressure cessation of blood flow occurs. The common criteria for diagnosis is: deep unresponsive coma, absence of brainstem function and reflexes and positive apnea test despite $\mathrm{pCO} 2$ being greater than $60 \mathrm{~mm} \mathrm{Hg}{ }^{2}$ In order to confirm brain death,irreversibility of such criteria has to be established along with absence of reversible etiology such as metabolic disturbances, neuromuscular blocking and toxic agents. Irreversibility can be proved with imaging techniques which demonstrate clear etiology or severe anatomical damage. ${ }^{3}$ There are particular circumstances when confirmatory tests come to play: (a) neurological assessment cannot be performed (e.g. due to severe maxillofacial injuries) (b) a primary metabolic/pharmacologic/endocrine abnormality (c) there is high cervical cord injury(d) there is diagnostic uncertainty due to spontaneous or reflex movements ${ }^{4}$ (e) When the pre requisites of brain death are not fulfilled and observation period has to be reduced (f)when children less than 5 years old are involved. ${ }^{1}(\mathrm{~g})$ early diagnosis is needed for organ transplantation.(h)hypothermia. In such situations other ancillary tests come to play. These either demonstrate absence of electrical activity or cerebral circulatory arrest. Methods to establish diagnosis of brain death include:

1) Nuclear medicine scans which show absence of both cerebral metabolisim and blood flow.

2 ) Transcranial doppler ultrasound

3) Electroencephalography and Evoked potential studies which demonstrate absent brain electrical activity. Both techniques may be inaccurate in comatose patients owing to drug induced hypothermia, intoxication and artifacts. $^{2}$

4) Catheter cerebral angiography: Angiography was regarded gold standard. Failure to detect intracranial flow is accepted as a confirmatory sign of brain death. ${ }^{5}$ However it is an invasive method, with complications such as final cessation of blood flow to remaining vessels and damage to transplantable organs. Catheterization of vessels requires expertise and shift of critically ill patients to cath lab could be challenging. Furthermore contrast agents could be artifactually introduced into intracranial circulation if injected under high pressure or if head is in dependent position. ${ }^{6}$

5) CT angiography and CT perfusion study: helps diagnose absence of cerebral blood flow

6) CT: Plain CT is ubiquitous, noninvasive, nontime consuming. CT findings in case of brain death are: diffuse hypodensity due to cerebral edema, decreased differentiation between grey and white matter, sulcal effacement, absence of basal cisterns, abnormally dense appearing arterial structures of circle of willis and deep venous structures mimicking contrast enhanced study which is due to intravascular stasis of blood or thrombosis reflecting absence of cerebral perfusion ${ }^{7}$, also termed as pseudo SAH. White cerebellum sign is when there is diffuse decrease in density of supratentorial brain with relative increased density of thalami, brainstem and cerebellum.

7) MRI, MRA, MRV: MRA is an accurate, non invasive, reliable technique in demonstrating the absence of intracranial flow. MRI findings in case of brain death are: diffuse gyral swelling, absence of flow voids, high signal on DWI with corresponding reduction in ADC poor differentiation of grey and white matter, transtentorial herniation.Other signs are bilateral transcerebral vein sign which is branching structures extending through cerebral hemispheres parallel or perpendicular to outer wall of lateral ventricle and bilateral cortical vein sign which is visualization of cortical veins of cerebral hemispheres. MRA and MRV will show no evidence of intracranial circulation in combination with non visualization of the intracranial veins. Factors such as cost, availability of imaging modalitiy and stability of patient for transfer are issues which may concern. Another issue is MRA is reduced sensitivity to slow blood flow ${ }^{3}$ which may mimic complete occlusion.

8) Other modalities: MRS and functional MRI. 


\section{CONCLUSION}

As radiodiagnostic studies and imaged cases of brain death are not very common there is a need to create its awareness and to exploit the usefulness of specific radiological findings of brain death. Plain CT and MRI are relatively easily available and provide ample amount of information and these can be used to arrive at a diagnosis of brain death than the invasive methods. The radiologist role will be to notify the clinician regarding the same which will help in decision making and avoid overburdening the patients family and its consequences to follow in present scenario.

\section{REFERENCES}

1. Wijdicks EF. Determining brain death in adults. Neurology. 1995;45(5):1003-11.

2. Jones KM, Barnes PD. MR diagnosis of brain death. American journal of neuroradiology. 1992;13(1):65-6.

3. Ishii K, Onuma T, Kinoshita T, Shiina G, Kameyama M, Shimosegawa Y. Brain death: MR and MR angiography. American journal of neuroradiology. 1996;17 (1):731-5.

4. Simpson P, Bates D, Bonner S, Costeloe K, Doyal L, Falvey S, Gaffin J, Howard R, Kane N, Kennedy CR, Kennedy I. A Code of Practice for the Diagnosis and Confirmation of Death.

5. Goodman JM, Heck LL, Moore BD. Confirmation of brain death with portable isotope angiography: a review of 204 consecutive cases. Neurosurgery. 1985;16(4):4927.

6. Monsein LH. The imaging of brain death. Anaesthesia and intensive care. 1995;23(1):44-50.

7. Eick JJ, Miller KD, Bell KA, Tutton RH. Computed tomography of deep cerebral venous thrombosis in children. Radiology. 1981;140(2):399-402.

Source of Support: Nil; Conflict of Interest: None

Submitted: 23-07-2019; Accepted: 13-08-2019; Published online: 21-09-2019 